Supporting Information for

\title{
Upper Rim Bifunctionalized cone-Calix[4]arenes based on a Ligated Metal Ion and a Guanidinium Unit as DNAase and RNAase mimic
}

Riccardo Salvio, ${ }^{* a}$ Stefano Volpi ${ }^{\mathrm{b}}$, Roberta Cacciapaglia, ${ }^{\mathrm{a}}$ Francesco Sansone, ${ }^{\mathrm{b}}$ Luigi Mandolini, ${ }^{\mathrm{a}}$ Alessandro Casnati. ${ }^{b}$

* To whom the correspondence should be addressed

riccardo.salvio@uniromal.it

\section{List of Contents:}

${ }^{1} \mathrm{H}$ and ${ }^{13} \mathrm{C}$ NMR Spectra of compound 3

${ }^{1} \mathrm{H}$ and ${ }^{13} \mathrm{C}$ NMR Spectra of compound 4

${ }^{1} \mathrm{H}$ and ${ }^{13} \mathrm{C}$ NMR Spectra of compound 6 S4

${ }^{1} \mathrm{H}$ and ${ }^{13} \mathrm{C}$ NMR Spectra of compound $\mathbf{1 a} 4 \mathrm{HCl}$ S5

${ }^{1} \mathrm{H}$ and ${ }^{13} \mathrm{C}$ NMR Spectra of compound 7 S6

Scheme S1 - Synthetic scheme for the preparation of compound 7 and 4. S7 
${ }^{1} \mathrm{H} \mathrm{NMR}$ of $\mathbf{3}\left(\mathrm{CDCl}_{3}, 300 \mathrm{MHz}\right)$
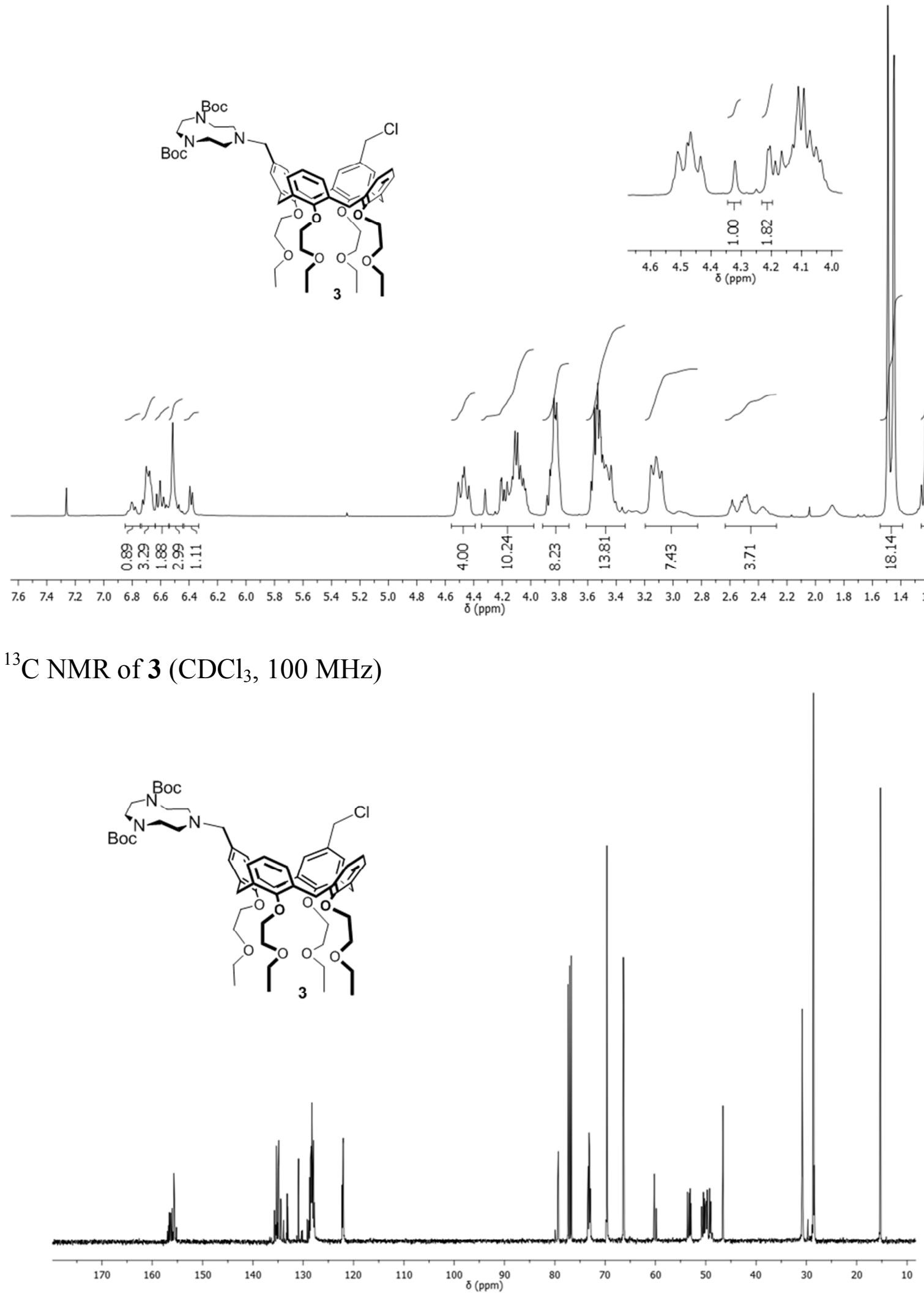
${ }^{1} \mathrm{H}$ NMR of $4\left(\mathrm{CDCl}_{3}, 400 \mathrm{MHz}\right)$

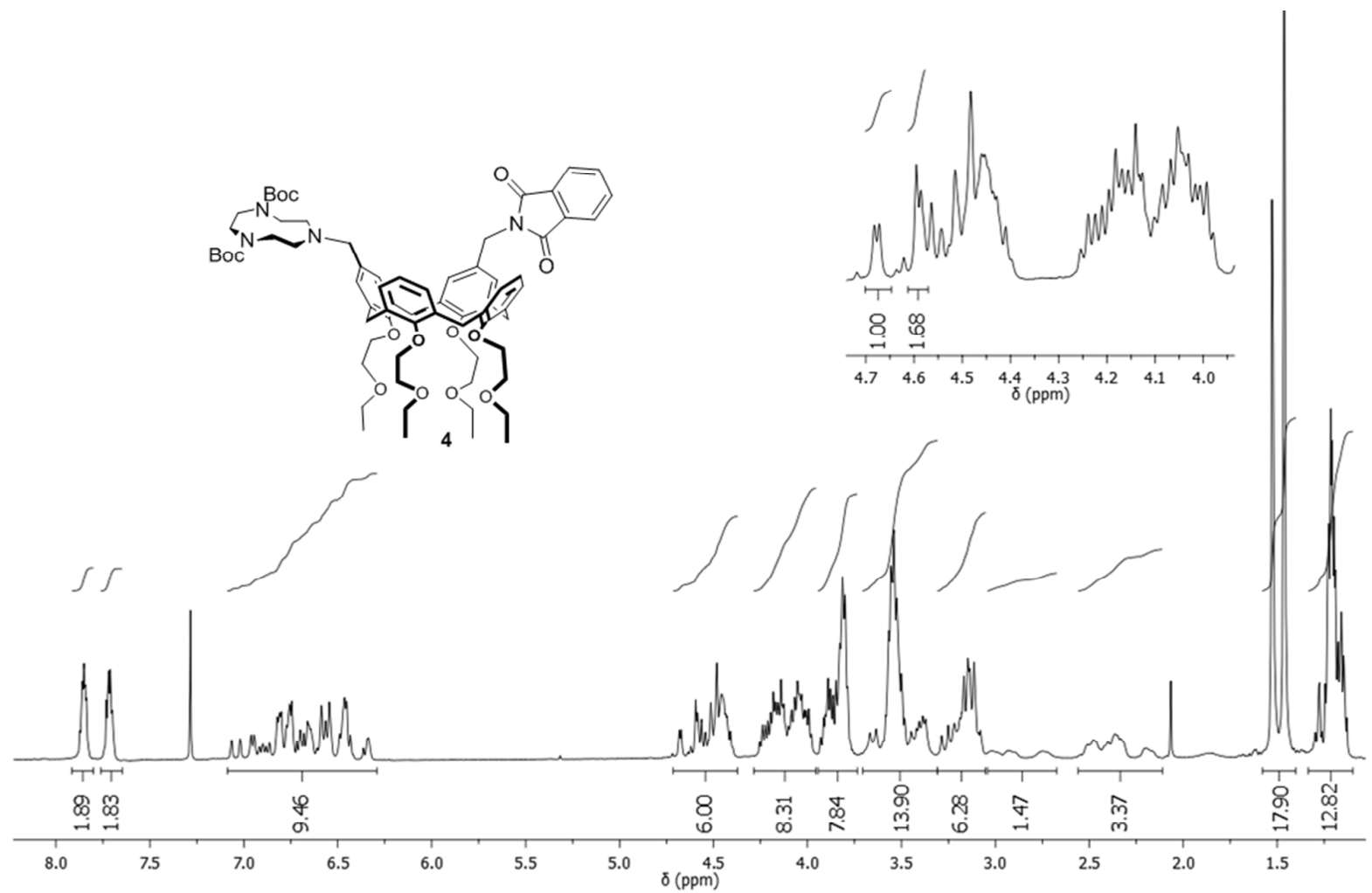

${ }^{13} \mathrm{C}$ NMR of $4\left(\mathrm{CDCl}_{3}, 100 \mathrm{MHz}\right)$

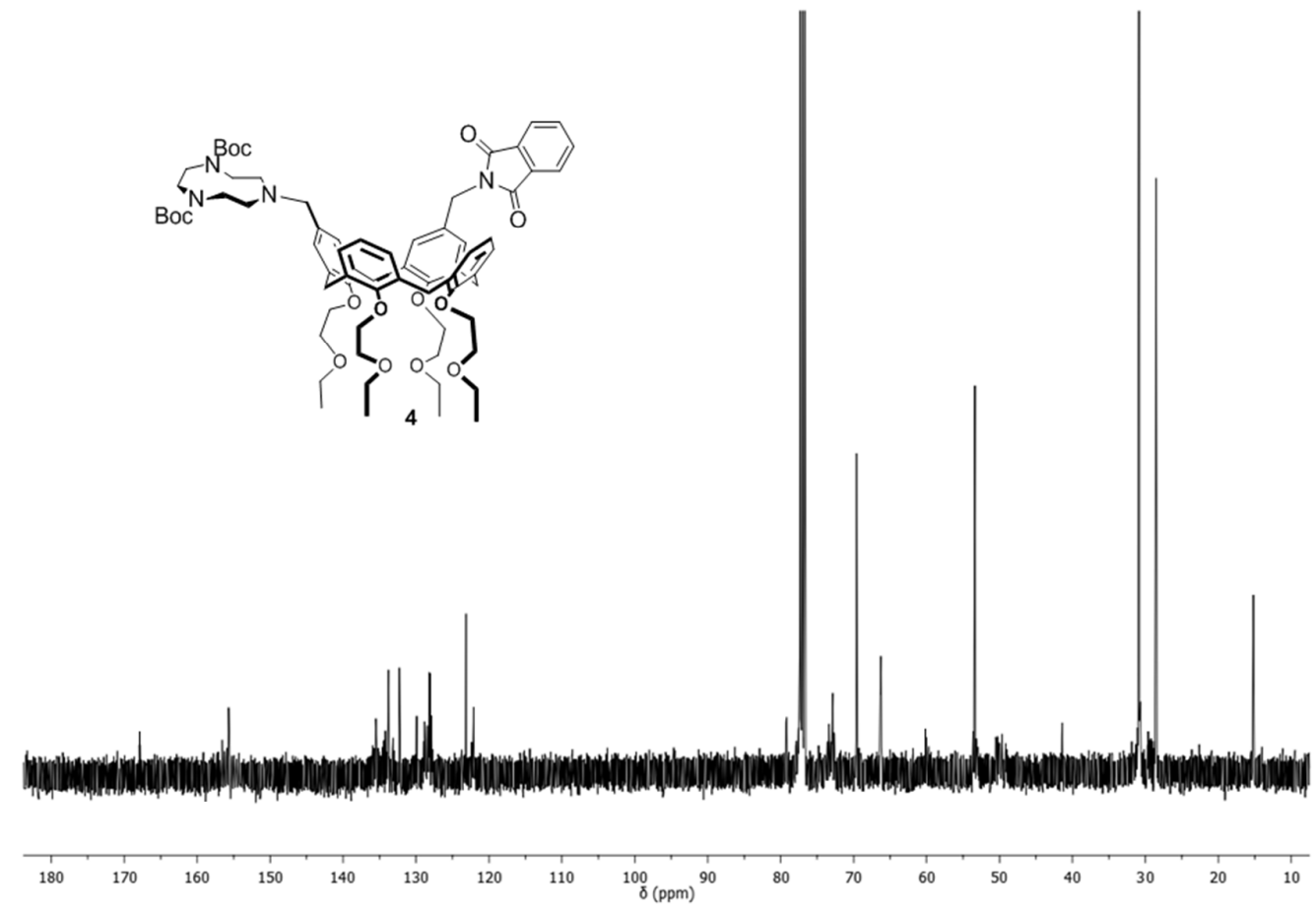


${ }^{1} \mathrm{H}$ NMR of $6\left(\mathrm{CDCl}_{3}, 400 \mathrm{MHz}\right)$
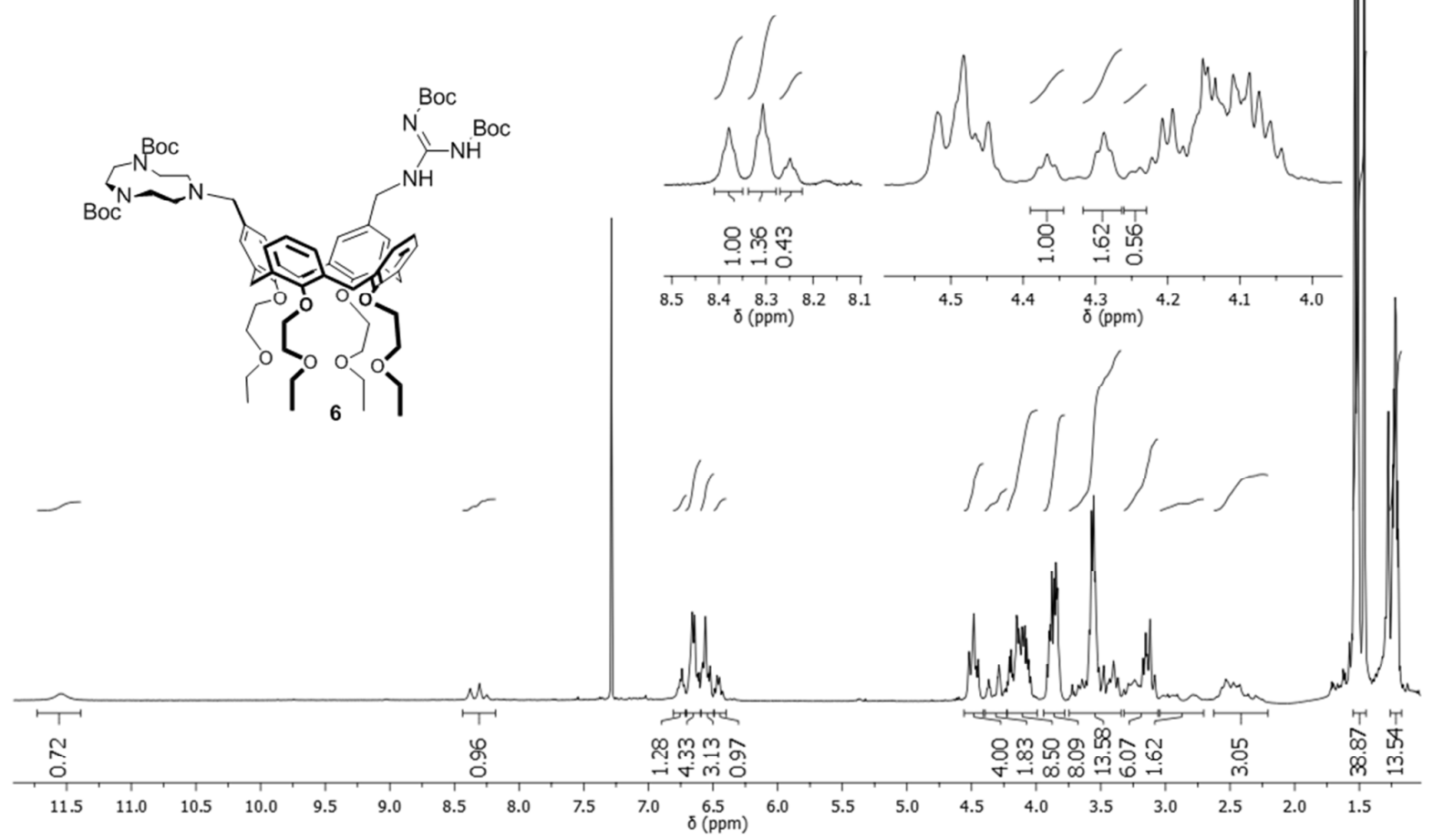

${ }^{13} \mathrm{C}$ NMR of $6\left(\mathrm{CDCl}_{3}, 100 \mathrm{MHz}\right)$
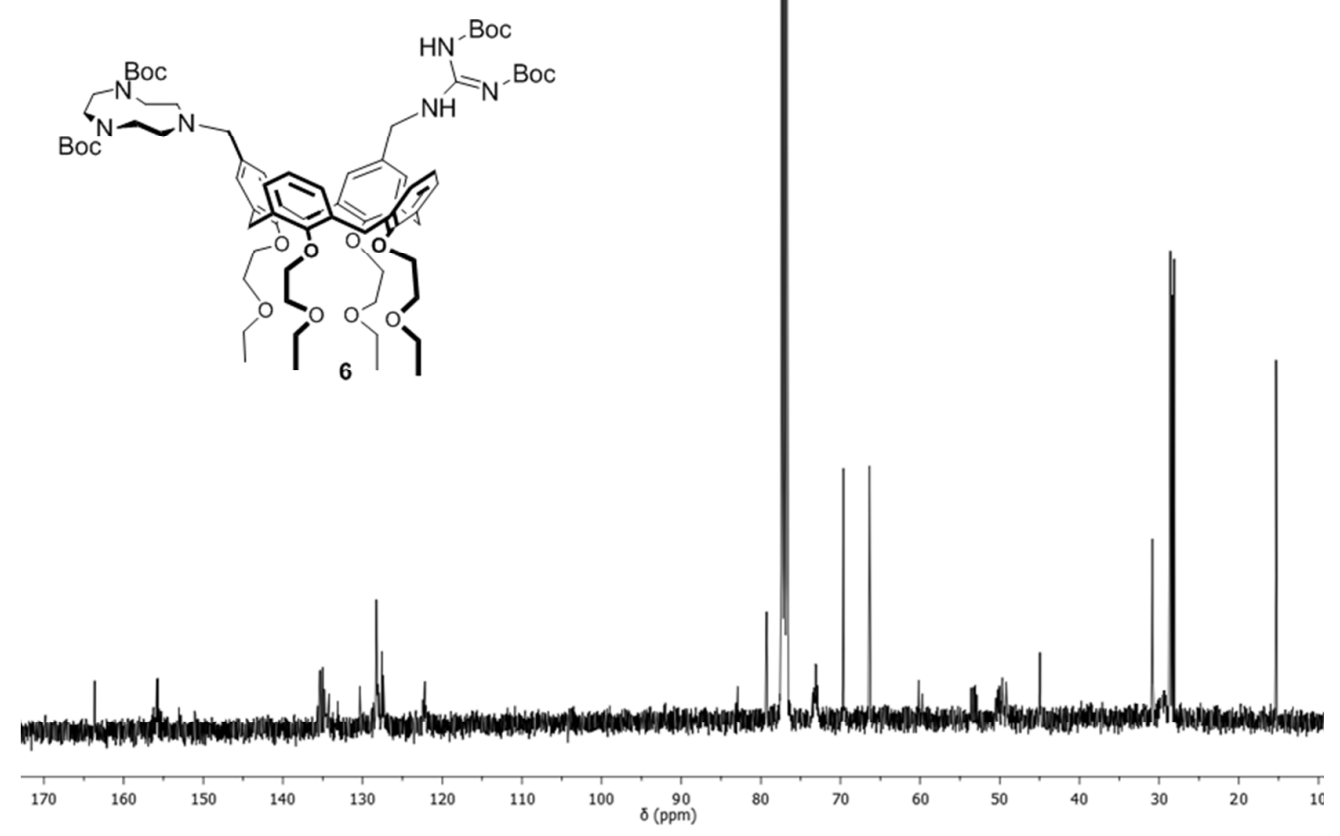
${ }^{1} \mathrm{H}$ NMR of $\mathbf{1 a}\left(\mathrm{CD}_{3} \mathrm{OD}, 400 \mathrm{MHz}\right)$

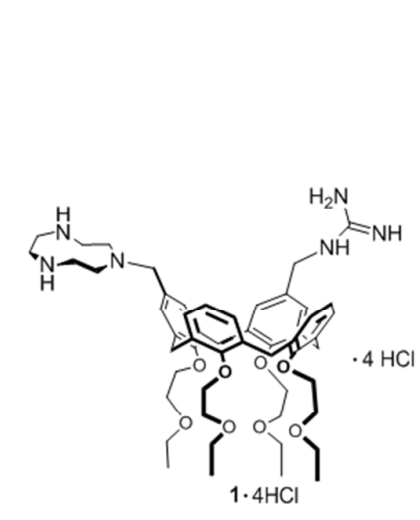

$\int \rho$
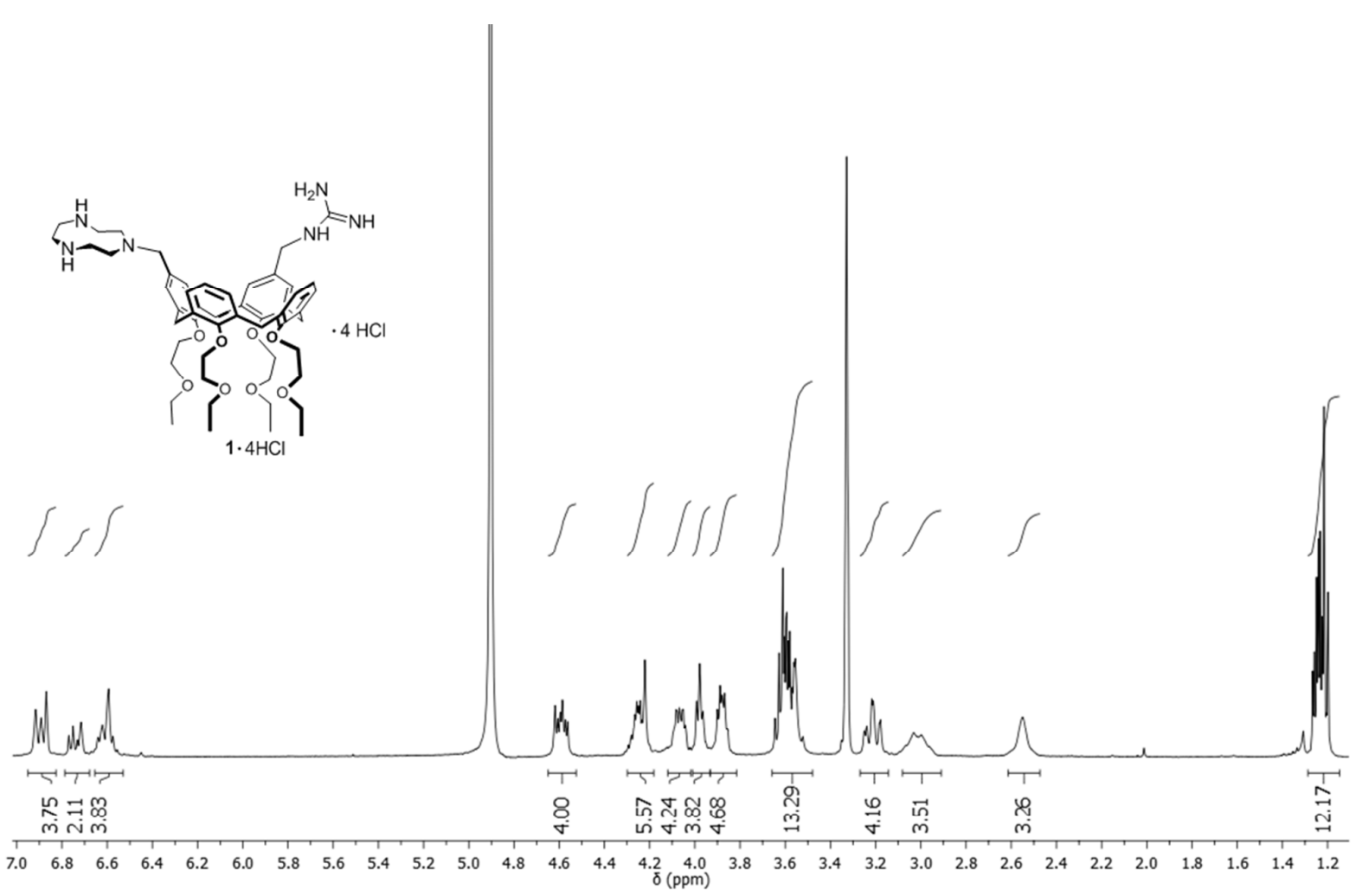

${ }^{13} \mathrm{C}$ NMR of $1 \mathrm{a}\left(\mathrm{CD}_{3} \mathrm{OD}, 100 \mathrm{MHz}\right)$
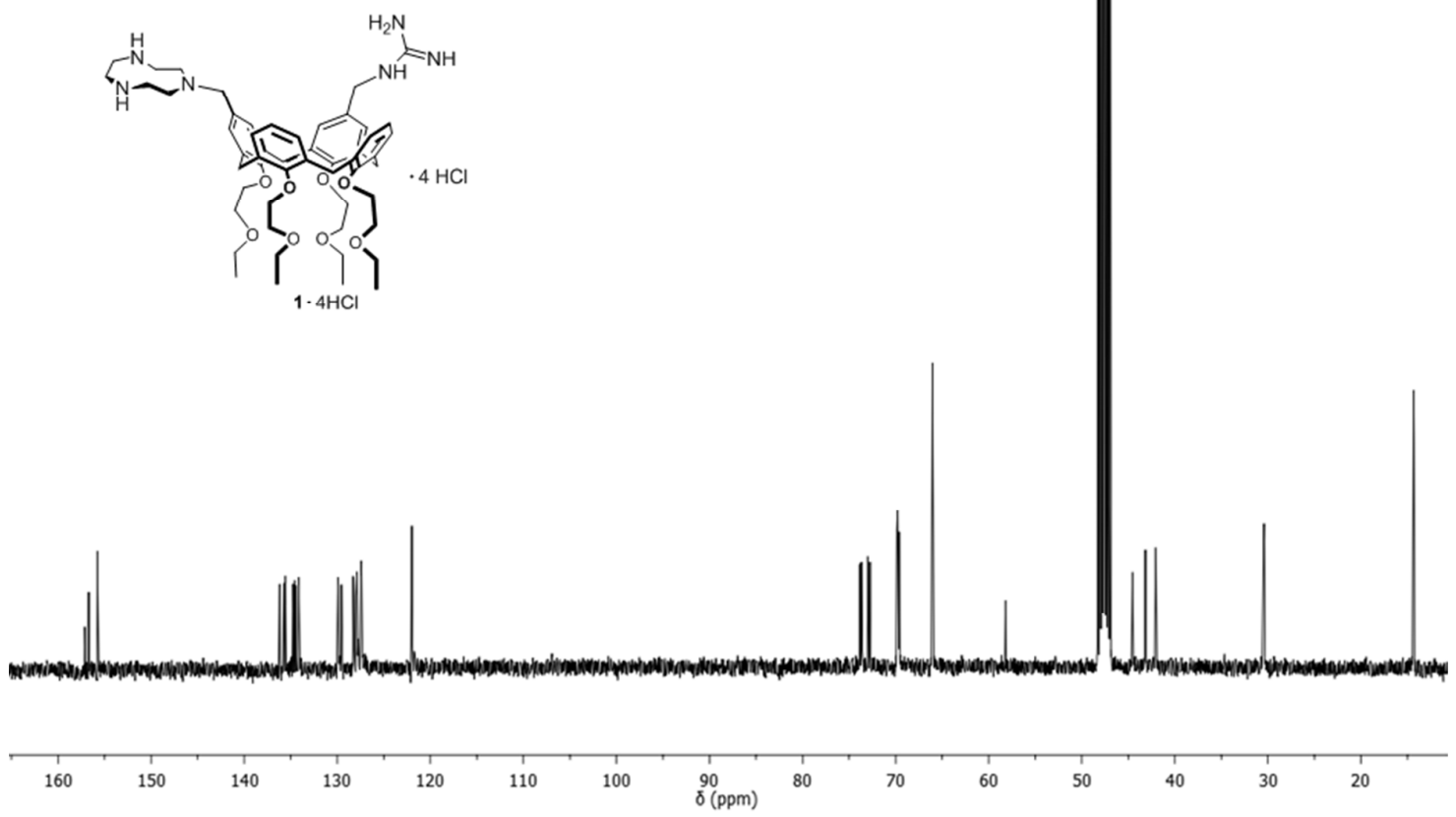
${ }^{1} \mathrm{H}$ NMR of $7\left(\mathrm{CDCl}_{3}, 300 \mathrm{MHz}\right)$

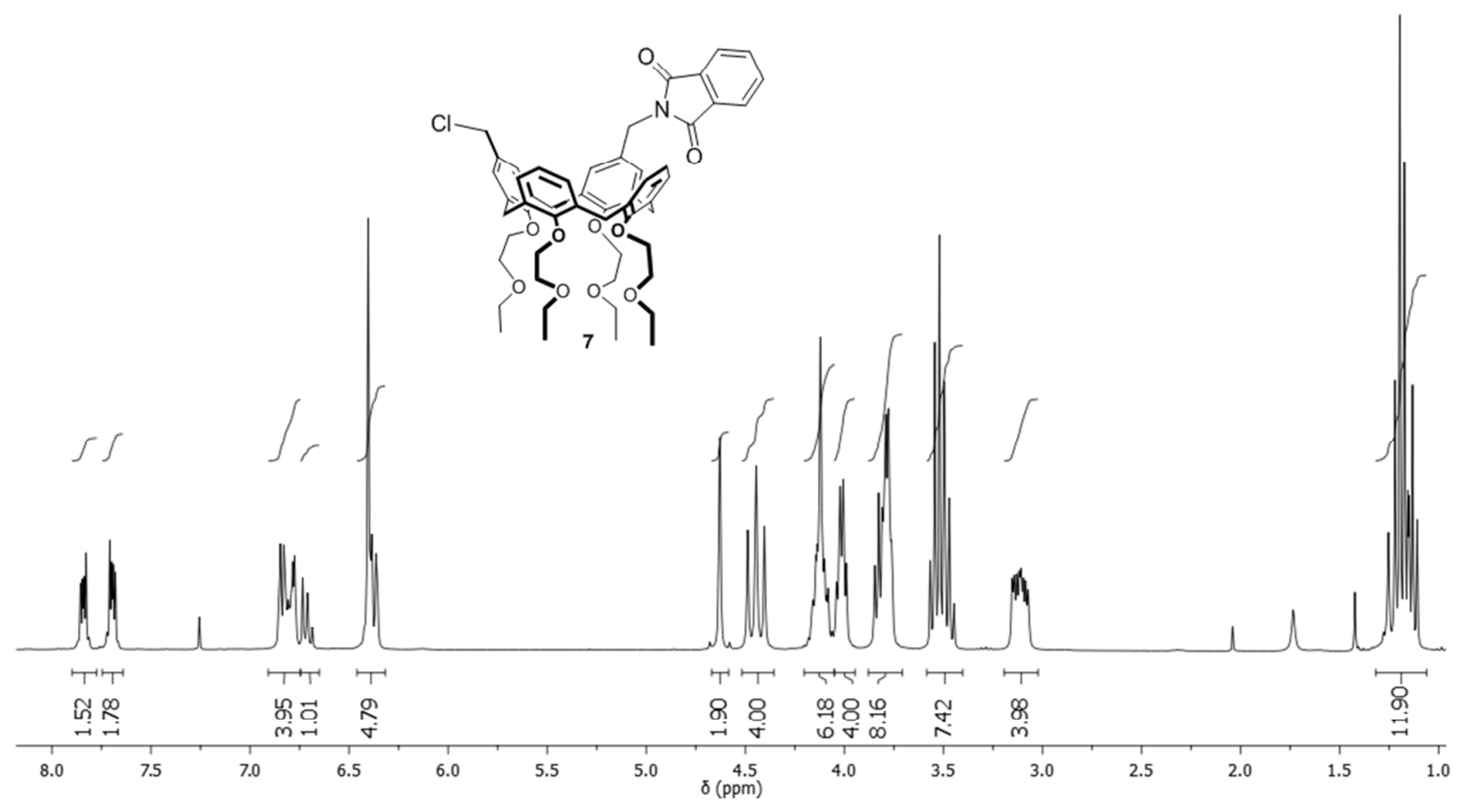

${ }^{13} \mathrm{C}$ NMR of $7\left(\mathrm{CDCl}_{3}, 100 \mathrm{MHz}\right)$
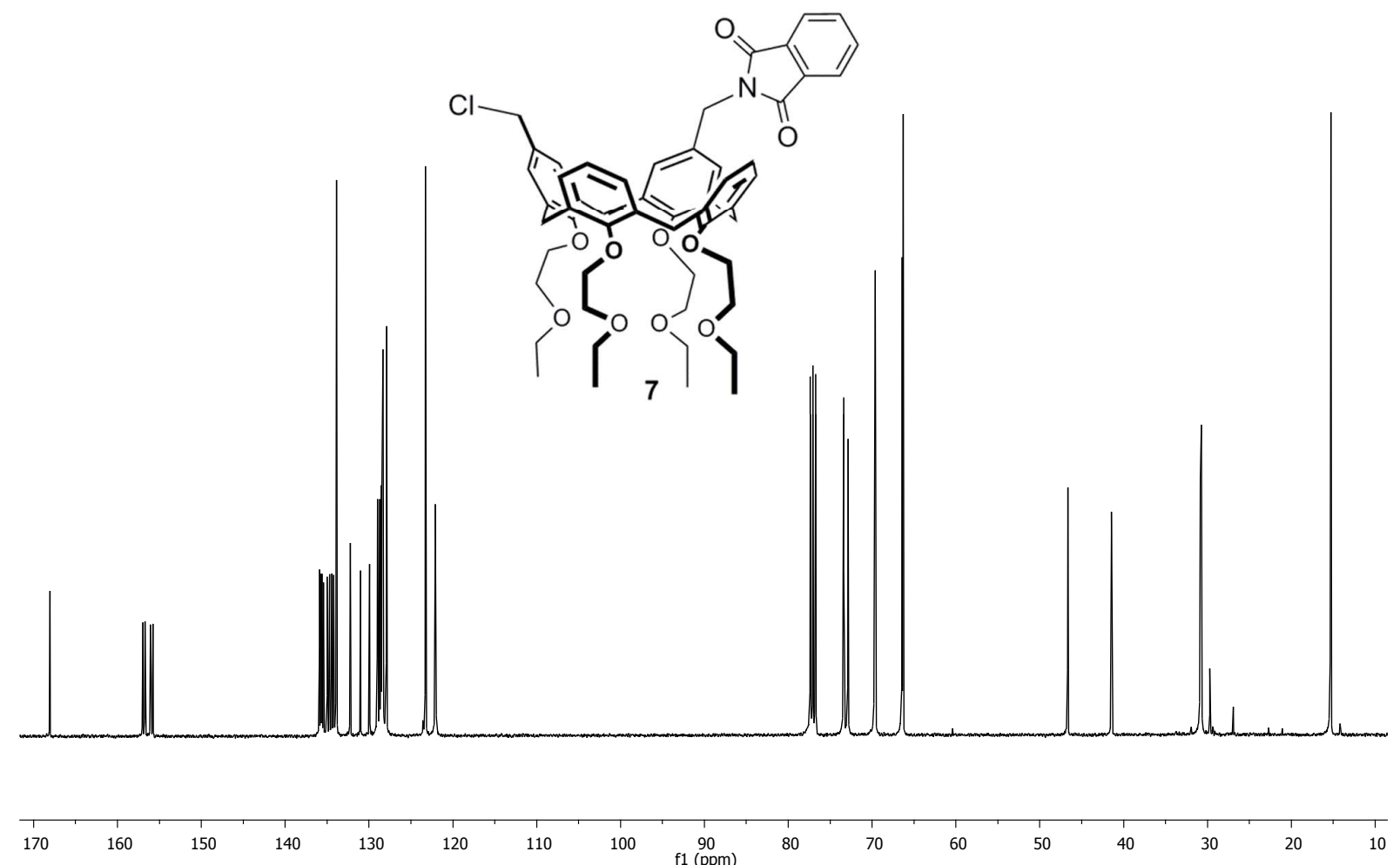

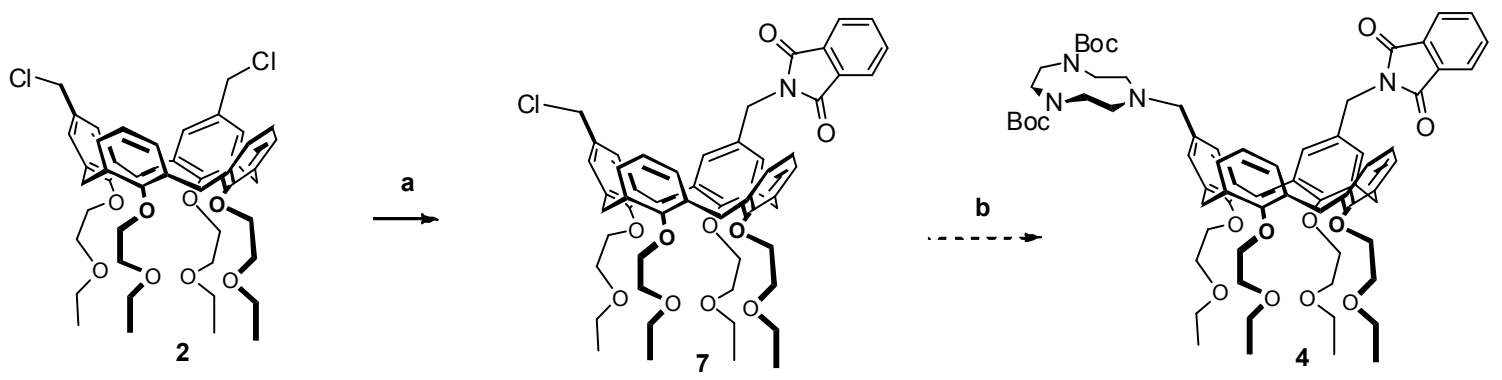

Scheme S1: a: potassium phthalimide; dicyclohexyl-18-crown-6, dry toluene, $110{ }^{\circ} \mathrm{C}$; b: $N, N^{\prime}$-bis(Boc)-1,4,7-triazacyclononane, dry $\mathrm{CH}_{3} \mathrm{CN}$, different bases, temperatures. 